\title{
MEASURING TRUST IN ONLINE SOCIAL NETWORKS The Effects of Network Parameters on the Level of Trust in Trust Games with Incomplete Information
}

\author{
Parvaneh Afrasiabi Rad, Svante Edzen and Soren Samuelsson \\ Department of Computer Science, Electrical and Space Engineering, Luleå University of Technology, Luleå, Sweden \\ Parafr-9@student.ltu.se, \{svante.edzen, soren.samuelsson\}@ltu.se
}

Keywords: Trust Level, Online Social Network, Trust Game, Incomplete Information, Network Parameters, Simulation.

\begin{abstract}
The most currently popular method for assessing trust in online social networks is Trust Game. The major studies in this area have established results formed into hypotheses for the effects of a number of network parameters on the extent to which individuals would place trust on each other. However, hypotheses for the effects of a few number of network parameters, such as Indegree, are not deducible since the restrictive game-theoretic assumptions that are imposed into the model do not let any such evidence available. To relax the game-theoretic assumptions, we develop a model for games with incomplete information, based on a game-theoretic model developed by Buskens (1998), and conduct a series of computer simulation of a model of Iterated Heterogeneous Trust Games (IHTG). We compare the results with those of Buskens' (1998) model and introduce Link-Strength as a new network parameter to investigate. Our results show a positive effect of both Indegree and Link-strength on the level of trust in a noisy environment. In addition, we come to a conclusion that current models can be fooled by the existing noise in the context of information transmission, such as inactive users in our case.
\end{abstract}

\section{INTRODUCTION}

The effects of the communication channel and the characteristics of interactions are the major interesting areas in CMC studies on trust in social networks (Riegelsberger, Sasse et al. 2003). In this respect, scholars strive to derive hypotheses for the effects of the structure of the communication channel, which represents the patterns of interactions, on the level of trust. The most currently popular method for assessing trust in online social networks is making use of "Trust Games" (Camerer and Weigelt 1988; Kreps 1992; Kreps 1996; Snijders 1996; Dasgupta 2000; Buskens 2002).

The game-theoretic model for measuring trust threshold developed by Buskens (1998) has been acclaimed to be the first model that provides hypotheses about both individual and global network parameters, in addition to deriving hypotheses about non-network parameters and their interaction effects with network parameters (Buskens 2002, chap 3). The model and analysis are applied for Iterated Heterogeneous Trust Games (IHTG). The outcome of the model suggests that network parameters influence the extent to which trustors would place trust on the trustee mainly through outdegree and density, whereas other network parameters have not been concluded to be influential on trust threshold (Buskens 1995; 1998; Buskens 2002, chap 3; Buskens and Raub 2008). However, such hypotheses have been driven based on a Pareto optimal equilibrium in trigger strategies for games with complete information. Such context requires postulating several assumptions and considerations in various aspects in developing the model, whereas most real situations are not governed by such circumstances.

Focusing attention, Buskens' (1998) gametheoretic model takes a counterintuitive assumption that the information is 'always and accurately' ( $p$. 286) passed from one entity to another in the network. The authenticity of the information, however, is not promised in social networks. Such facts that oppose the assumption of the reliability of the information are referred to as 'noise' (ibid, p. 286). Refusing to incorporate the noise in the context of information transmission due to the restrictive game-theoretic assumptions, has led this model to be unable to derive hypotheses about the learning effects of embeddedness on trust, hence 
leaving network parameters such as indegree as uninfluential on the level of trust (ibid). In addition, the game-theoretic assumptions impose some circumstances to the context of information transmission which is far from the realistic environments. To extend the game-theoretic model, so that predictions about the effects of network parameters in the context of noise can be derived, we should relax a number of selective assumptions.

In this study, we make assumptions about incomplete information, and the existence of noise in the context of information transmission. The new context would let us investigate the effects of additional network parameters, i.e. Indegree and Link-strength. It is also closer to the context of real social networks, in the sense of both assumptions and structure. We boost the influence of the circumstances of incomplete information by taking sample networks that are very large in size, in order to conceal the structure of the network from the players. Simulations are run on 6 networks that are sampled from Youtube, for their structure to be closer to reality. The results are further analyzed to derive hypotheses about the effect of a new set of different network measures, indegree and linkstrength, on the level of trust in the context of noise. We utilize the game-theoretic model, developed by Buskens (1998), for its validity and make alterations to its assumptions to form a new context.

The following section starts with the framework of this study by introducing the sources of noise and its effects. It also includes details of the model that is developed in this study with the assumptions of games with incomplete information. It follows with a presentation of the simulation method in addition to the results of the regression analysis of the simulated data. The hypotheses driven from the analysis of the results can be found at the end of that section.

\section{THE MODEL}

As it has been previously discussed, the gametheoretic model does not circumstantiate intuitive hypotheses as far as indegree is concerned. Aside from indegree effects, noteworthy is the reason for such, which is due to the assumption that the information about the trustee's behavior is, always, positive and accurately transmitted in the network. After all, the role of indegree is more conclusive in a "noisy" environment (1998, p. 286; Buskens 2002, p. 90). A trustor could be reluctant to sanction a trustee if she obtains information about the abuse of trust form one trustee and she cannot verify the information herself. She will decide to execute sanction only in case she receives such negative information repeatedly. The extent to which trustors receive information in a network, indegree, thereupon will have an effect on trust (Buskens 2002, chap 3). Regardless of the ways different trustors could interpret incoming information about the trustee's behavior to further forward it to the next trustor in the game, it is reasonable to conclude that, in the context of noise, a trustor with larger indegree is more certain about the accuracy of information in hand by virtue of obtaining information from multiple sources (Buskens 1998). Also, the positive information about the behavior of the trustee is more reliable when it is transmitted through such trustor. Accordingly, an inactive user i.e. an actor with a large indegree and a small outdegree, is a source of likely enough reliable information, while not contributing to the flow of information in the network.

We do not aim to manipulate the game-theoretic context, deviate from trigger strategies, alter the equilibrium or introduce a new one. Notwithstanding, we will assume that subsisting information about the behavior of the trustee is not always considered to be accurate and neither is perfectly transmitted between trustors. The origin of such information, the amount and the order and structure of its transmission is not a matter of concern. Trustors, indeed, follow trigger strategies to decide upon placing trust, however are triggered not merely by receiving information about the abuse of trust from the previous trustor, but as they are "infected" by the information that they receive. These arguments are valid only in the context of a an information diffusion model that incorporates the idea that 'an actor does not receive information as a package relinquished by the sender, but rather is "infected" by the information given to him' (Buskens and Yamaguchi 1999, p. 5). Therefore, the information can be considered as unreliable not only due to inaccuracy, but also resultant from misinterpretation, information distortion during transmission, etc.

\subsection{Assumptions}

Buskens' (1998) game-theoretic model proposes that Outdegree and Density are two network parameters which predict almost all variance that could be attributed for the trust threshold. These two factors are weighted by the parameters of the game and considerations of the equilibrium. Still, to satisfy the 
assumption that users are triggered by the impact of information, it is important to find out to what degree they are infected. The answer to this question cannot be explained only by Outdegree and Density, since the amount of information obtained does not mean that the receiver is certainly affected by it. In order to estimate how much a piece of information can infect a user, we include an additional network parameter, namely Link-Strength, into the model to measure the strength of a tie between two trustors. If the relationship between two trustors is strong, one can be influenced even with the lowest amount of information obtained from the other. In other words, users with some close friends, which are characterized by strong friendship links, are more likely to influence (or be influenced by) them than those who hold many friendship bonds but almost no close friends, provided that the two groups create comparable amounts of content. So, the value of Link-Strength can raise the effects of Outdegree and Density on trust threshold. Here, we suggest for the strength of a tie to be defined as the average number of two-way interactions between two actors that are connected by that tie. Link-Strength, $S(i, j)$, is positively related to the number of incoming and outgoing interactions between two nodes of $i$ and $j$.

$$
S(i, j)=\frac{a_{i j}+a_{j i}}{D_{\text {out }}(i)+D_{\text {out }}(j)}
$$

\subsection{Solution of the Model}

As the first step to incorporate the effect of noise in information transmission into the model, we inspect the model for the way inactive users would affect measuring trust threshold. These actors barely send any information out and are not as influential as others in information transmission. However, the network parameters that are assigned to them can still fool the model and result in a higher level of trust including inactive users in the calculations. An extreme of such actors are those who have a high Indegree together with a negligible Outdegree value. Thereupon, to assign a zero value to the trust threshold around all inactive users, the value of $\vartheta_{i}$ is multiplied by the hyperbolic tangent of their Outdegree value. In such a way, in case the outdegree is equal/close to zero, the trust threshold will be equal/close to zero.

$$
\vartheta=\left\lceil\tanh D_{\text {out }}\right\rceil *\left(k \rho_{1} D_{\text {out }}+k^{2} \rho_{1} \rho_{2} \Delta\right)
$$

In addition, a major concern for measuring the learning effects of network embeddedness is that under the assumptions for the games with incomplete information, the impact of the control effects of embeddedness on the trust threshold is lessened to a considerable degree (Buskens 2002, chap 3). The reason is that in that situation, no sufficient cues from the network are provided for the trustee to control his behavior by a sanction probability or a bad reputation aftermath. For that reason, we propose to diminish the role of control effects in the game-theoretic model and focus on the impact of information diffusion. In this manner, the parameters of the game will be defined as constant in our model in order not to be influential on the variation of the level of trust.

Moreover, for the sake of incomplete information, the network structure is assumed to be limited in the eyes of the trustee. This is implemented by introducing networks with a large number of nodes, the structure of which seems extremely far from a trustee's perception and is unknown to him, except a few close relations.

\section{SIMULATION}

To achieve findings applicable to heterogeneous networks, in which the assumptions for games with incomplete information are applicable, we use a simulation method. It is worthwhile to recall that, in the model developed in this study, no specific trustee is identified. In fact, the trustee is considered to be the one with whom a trustor has interactions.

\subsection{Sampled Networks}

Earlier, in the development of the model, the size and structure of the sample networks have been contemplated in order to lead to a situation closer to the assumptions for incomplete information. Knowing that the results of Buskens' (1998; 2002, Chap. 3) game-theoretic model has shown no effects of network size on the trust threshold, we feel free to decide upon the number of nodes in the network. Building the networks that would form the basis for the simulation requires an exhaustive investigation of many factors. Knowing that many methods for creating sample networks carry considerable drawbacks, we have decided to create networks for simulation scenarios by sampling from an existing online social network, Youtube. The only concern is to fetch a number of network structures, representing an actual social network, for simulation scenarios. Networks are sampled starting from a randomly selected user with an active profile and large number of friends, using snowball sampling (Goodman 
1961; Salganik and Heckathorn 2004). The algorithm is provided with a random video ID published by a user whose friends are added to the network with the same structure and connections. To do so, the Youtube network is crawled with a snowball method to find them.

Sampling networks resulted in several networks with thousands of nodes, among which 6 networks with the number of users between 10,000 and 19,800 have been selected on which the simulation is to be performed.

\subsection{Experimental Design}

Each of 6 abovementioned networks constitutes a scenario for which the network parameters are computed in the simulation. The values of Outdegree, Indegree, Density, and Link-Strength are calculated for every node in the network. These values are further regressed on the values of Indegree and Link-Strength to conclude the influence of Indegree and Link-strength on trust threshold in the context of noise. The (Spearman) correlation coefficient between Indegree and LinkStrength equals 0.093 in average for all 6 scenarios which is low enough to make us confident to perform their regression analysis separately. However, the large number of cases in each scenario is sufficient to distinguish the effects of the different network parameters. For each node, two values of trust threshold are calculated: model 1, and model 2 . Model 1 is the same as the solution introduced by Buskens (1998; 2002) for the game-theoretic model. The latter is the value of trust threshold after eliminating inactive users from the solution due to the insignificance of their role in information diffusion. The two models will be compared to make deductions regarding additional networks parameters in this study.

Both network and non-network parameters have to be sampled for each simulation scenario. Network parameters are calculated for each node in every sampled network. Non-network parameters, on the other hand, follow the same variation that is used by Buskens (1998; 2002) and sampled independently (in the probabilistic sense) for each network. Noteworthy here is that, in each scenario, the value of the game parameters that are involved in the calculations of the trust threshold is set to be the same for all trustors in a network. The reason is to prevent its variation from being considered to be effective in the calculations. This is perfectly in line with the fact that introducing the assumptions of incomplete information into the contexts of IHTG would reduce the control effects of network embeddedness (Buskens 1998; 2002) that are implemented by the game parameters in this model.

The simulated system is a social network, demonstrated by its graph with finite number of nodes, for each the dependent variables are computed to generate the simulation data. The simulation environment is developed using Java and Java Universal Network/Graph Framework (JUNG) (2009). A "terminating simulation" (Banks, John S. Carson et al. 1996, chap 12) is performed for each scenario, as the termination circumstances for each run is embedded in the simulation scenario description. Each simulation scenario starts traversing the network graph from a node to compute the required values for network parameters and trust threshold for both models, and terminates when the computation is done for the last node in the network. The system is studied for a single point of time at which the network is sampled from Youtube, hence assumed to be in a constant state during the simulation. The outcome of the simulation is a set of random values that constitute the "simulated data" for further analysis. Here, the output consists of two network parameters, Indegree and Link-Strength, in addition to the two dependent variables of trust threshold for both models 1 and $2, \vartheta_{M 1}$ and $\vartheta_{M 2}$ respectively.

\subsection{Analysis of the Simulated Data}

The values for the dependent variables in each data set do not fall below zero and even though they do not always take a known value, they are known to be elements in an interval. Thus, a regression analysis of the dependent variables can be performed. However, we cannot perform a linear regression since the values of variables do not follow a normal distribution. Therefore, to determine the correlation between two variables, a Spearman regression analysis is applicable (Sheskin 2004, p. 1360-1362). In addition, to make sure if the output values from the simulation are valid to be further analyzed, we perform a confidence level t-test on the dependent variables. The results show that after the first simulation run the error in the average of the trust threshold would not be more than $5 \%$ with the $95 \%$ confidence, and with repeating simulation for 4 times we can be confident that with the probability of $98 \%$, the error would not exceed 2 percent.

Table 1 shows the results of the Spearman regression analysis of the effects of Indegree and Link-Strength on the trust thresholds for both models. Spearman R-squared value for the 
regression is given for each model in every scenario. The second raw in the tables represents the hypotheses on the effects as are derived from the analytic results. The results obtained from the regression, either strong or weak association between the network parameters and trust thresholds, are significant for all simulation scenarios $(p<0.0001)$.

Table 1: rho of the Spearman regression of Indegree and Link-Strength with trust threshold in both models for all six scenarios $(p<0.0001)$.

\begin{tabular}{|c|c|c|c|c|}
\hline & \multicolumn{2}{|c|}{ Indegree } & \multicolumn{2}{c|}{ Link-Strength } \\
\cline { 2 - 5 } & Model 1 & Model 2 & Model 1 & Model 2 \\
\hline Hypothesis & + & $?$ & + & $?$ \\
\hline Scenario 1 & 0.47 & 0.04 & 0.40 & 0.25 \\
\hline Scenario 2 & 0.58 & 0.08 & 0.40 & 0.23 \\
\hline Scenario 3 & 0.52 & 0.05 & 0.39 & 0.23 \\
\hline Scenario 4 & 0.56 & 0.04 & 0.40 & 0.22 \\
\hline Scenario 5 & 0.50 & 0.05 & 0.41 & 0.26 \\
\hline Scenario 6 & 0.52 & 0.04 & 0.37 & 0.24 \\
\hline
\end{tabular}

The results of the effects of indegree on trust threshold in model 1 show a moderate positive correlation between two variables, meaning that the value of the dependent variable, trust threshold, increases in the Indegree of the trustors. This also denotes that introducing noise in information diffusion would result in a context in which conclusions about the effects of Indegree on trust threshold can be driven from the game-theoretic model. Moreover, the results of Model 1 for Indegree support the previous finding of Buskens (1998) showing that 'for given outdegrees, the network structure centralized with respect to indegree around the buyers with the highest outdegree is the structure for which all buyers have the highest trust threshold' (p. 277). Considering that Indegree is not an element of the computations for trust threshold in the models, as it is calculated based on the Outdegree and Density, however, it cannot be claimed that the values for Indegree and Outdegree are independent from each other, since for a network as a whole, the aggregated number of indegree equals to that of outdegree.

In the second model, users with high values for Indegree and Density do not necessarily contribute to the trust threshold, unless having an Outdegree value equal to at least 1 . The considerable correlation between the trust threshold and Indegree drops dramatically to about zero after eliminating the effects of inactive users from the computations for trust threshold. The same fall is conspicuous in the average trust threshold in each network, since its value is set to be zero for inactive users. This represents a roughly extreme case in which inactive users can fool the model to return a fair value for trust threshold as of their ample Indegree value, whereas the actual trust threshold is less for those users because of their latent contribution to information diffusion.

According to the findings on the effects of LinkStrength on trust threshold, it is reasonable to infer that Link-Strength is probably an important factor that should be considered in the analysis of social networks in case of studying the learning effects of network embeddedness. The results of the regression in model 1 show a fair positive correlation between the two variables so that about 39 percent of growth in the trust threshold can be explained by the value of Link-Strength. Even after removing the effects of users with high Indegree value paired with a zero Outdegree, this association falls to $25 \%$, in average, which is not weak enough to be completely neglected. Such inference is intuitively justifiable. In a noisy environment, if the relationship between two trustors is strong, one can be influenced by even the lowest amount of information received from the other. Ergo, the information flowing between trustors who have some close friends, i.e. distinguished by strong connection links, are more likely to be influential than between those who have many friends but almost no close ones. This is indeed the case under the circumstance that both groups transfer comparable amounts of information.

The rate at which the Spearman's rho falls after inactive users are dismissed from the model 1 is shown to be significantly higher for Indegree than it is for Link-Strength. Intuitively speaking, an explanation of such can be that inactive users do not make strong friendship relations, thus the elimination of those would not ensue omission of a considerable number of strong links. Therefore, the value of network parameters will not experience a prodigious change in regards to the Link-Strength, so its effects on the trust threshold will still remain roughly the same. However, a reduction of those effects is reasonably predictable. Furthermore, such variation in the drop rates of rho values of Indegree and Link-Strength can be interpreted so that the conclusions for the effects of Link-Strength on the trust thresholds are more reliable. Of course, we make such statement under the circumstances that the values of Indegree and Link-Strength are considered to be calculated with independent network elements.

\section{SUBSTANTIVE IMPLICATIONS}

The assumptions of the model in this study results in 
diminishing the control effects of network embeddedness, while altering the focus of the gametheoretic model to the learning effects, influenced by the role of information diffusion between trustors. The model applies the previous findings, and the results extend theoretical hypotheses for trust in trust relations, and are in accordance with the existing literature (Raub and Weesie 1990; Coleman 1994; Weesie, Buskens et al. 1998).

The following hypotheses express the outcomes of the model:

Hypothesis 1 . In a context with noisy information, trust increases with the value of Indegree of the trustors.

Hypothesis 2. In a context with noisy information, trust increases with the values of Link-Strength of the trustors.

Hypothesis 3. In a context with noisy information, the positive effects of Link-Strength on trust are more promising and unyielding than those of Indegree.

Hypothesis 4. In a context with noisy information, the high Indegree value of users who do not supplement information diffusion in a network do not lead to an increase in the trust that can be placed.

Figure 1 illustrates the driven hypotheses in the context of this study, while the previous hypotheses for network parameters still remain valid. It also shows the position of our model, and its assumptions, related to Buskens' (1998; 2002) game-theoretic model.

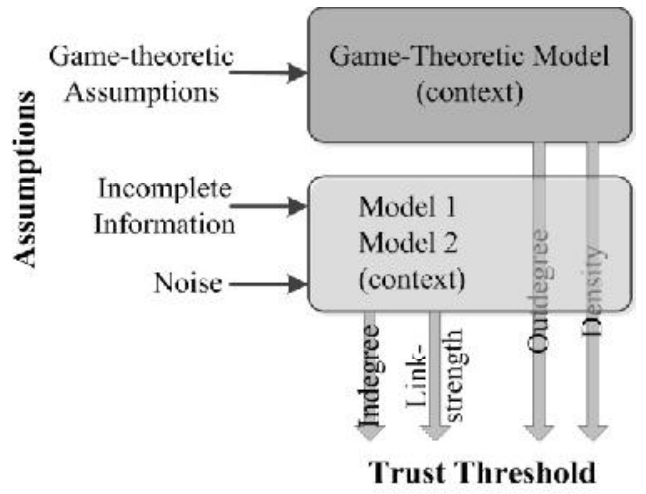

Figure 1: The assumptions and outcomes of the model for games with incomplete information.

\section{CONCLUSIONS}

The results of this study extend the findings of cases with complete information, while those findings remain valid in the newly developed context. The hypotheses for two network parameters, Indegree and Link-Strength, could not be driven in a network of trustors where information is assumed to be accurately transferred between Trust Game players. Adding the possibility of existing corrupt pieces to the flow of information in a network creates an environment that is closer to reality in which learning about the behavior of the trustee is more complex and affected by more parameters. Particularly, games with incomplete information are to be utilized for analyzing the learning and control effects of embeddedness in an integrative manner. However, in respect of theoretical modeling, relaxing game-theoretic strong rationality assumptions and introducing more realistic ones about how actors use relevant information that they obtain, seldom can come to a balance with analytic tractability. In addition to the intricacy of models with more realistic assumptions, knowledge about what realistic assumptions could be is limited because the effects of learning and control mechanisms has not yet been successfully cleared up by empirical researches (Buskens and Raub 2008). Corresponding to the situation, we have tried to relax less disturbing assumptions and introduce a few ones regarding to the games with incomplete information to be able to extract results for the learning effects of network embeddedness on the level of trust.

We have shown that trustors with higher Indegree have the capability to certify the positive information about a trustee while they receive wide variety pieces of information about others' experiences with the trustee. The assumptions of this model approximate those of the contagion models for information diffusion in heterogeneous networks (Buskens and Yamaguchi 1999) rather than the assumptions in transit models for such (Friedkin 1992; Yamaguchi 1996). Contagion models measure the extent to which an individual is influenced by information that is flowing in a network, whereas in transit models it is sufficient for an actor to obtain the information to be affected by it. The unrealistic assumptions of the transit models let them overestimate the effects of a number of network parameters. Here, we argue that not all the values for network parameters are conclusive and cannot be considered as effective on the trust level. We suggest that the Outdegree value should be weighted by the strength of the links through which information is transmitted between two actors, so that it would be possible to conclude the extent to which the information in flow is actually influential.

To link these results to the discussions on the control and learning effects of embeddedness, it can be concluded that a piece of negative information 
about the trustee per se, and the opportunity for the trustor to exit the iterated trust games, does not promise additional control opportunities for the trustor in this model. Besides, adding the opportunity of spreading "voice" in a network creates more learning as well as control possibilities for the trustors. This is in agreement with the assertion by Buskens (2003) that '... the two aspects of voice [control and learning] need to be combined by the trustors to enable more trust in the trustee' ( $p$. 246). Such a result can be in favor of the situations in which trustors can sometimes experience negative outcomes while the trustee has not intentionally abused trust (for examples, see Radner 1981; Porter 1983). Learning is expected to be more important in such situations where a piece of negative information about trustee's behavior, solely, should not fundamentally ensue in an exit option. Another such situation is one where the trustee does not have a fixed type, thus the trustors would hesitate to exit and more like to observe the changes in the trustee's type. Therefore, every experience with the trustee would be worthwhile to the trustors (Mailath and Samuelson 2001).

This model also carries some restrictions. Even though we have minimized the effects of the game parameters in the model to be able to aim the attention at the effects of information flow between trustors, the trustors are still considered as playing successively with the trustee. Therefore, we cannot claim that the model can be applied to the situations in which trustors can play simultaneously with the trustee. The simulation study context that we have implemented in this study can be considered as one in which the order of the Trust Games is not a matter of importance (see Buskens 2003), however, no assumption is made in this regard. Another important disadvantage of this model is that trust is not investigated joined together with distrust. In fact, it is reasonable to deduce that the existence of an amount of negative information on the trustee's behavior could take a few more steps than just reducing the trust level and ensue distrust. Measuring distrust, involves different factors while the relative assumptions are still ambiguous since the topic has not yet attracted enough attention of scholars. Still, extended assumptions, as mentioned above, would modify the model to one that is closer to real situations.

Certainly, the discussions on how such assumptions can be changed or extended encompass a wide range of considerations. However, in this respect, Buskens (2003) states that ' $\ldots$ I think that we lack considerable knowledge about what actually reasonable assumptions are especially related to information availability of actors, information exchange, among actors, and how actors actually use this information [to update their beliefs, or decide upon sanctioning the trustee] ...' (p. 247). Therefore, it would be fruitful to develop such experimental designs that allow for testing both the implications of theoretical models and the way actors use the information obtained while playing a game. For studying the learning effects, it is more favorable to analyze the decision making process of actors rather than the decision itself (ibid). Such contemplative propositions would extremely add to the complexity of the current models of Trust Games and can form cases for further research.

\section{ACKNOWLEDGEMENTS}

Clarifying conversations with $\mathrm{PhD}$. Saeed Dastgiri, professor at Tabriz University of Medical Sciences, Iran, and $\mathrm{PhD}$. Ali Ardalan are gratefully acknowledged.

\section{REFERENCES}

Aberer, K. and Z. Despotovic (2001). Managing trust in a peer-2-peer information system, ACM.

Artz, D. and Y. Gil (2007). "A survey of trust in computer science and the semantic web." Web Semantics: Science, Services and Agents on the World Wide Web 5(2): 58-71.

Banks, J., I. John S. Carson and B. L. Nelson (1996). Discrete-Event System Simulation, Prentice-Hall International, Inc. .

Beth, T., M. Borcherding and B. Klein (1994). "Valuation of trust in open networks." Computer SecurityESORICS 94: 1-18.

Bonatti, P., C. Duma, D. Olmedilla and N. Shahmehri (2005). An integration of reputation-based and policybased trust management. The Semantic Web Policy Workshop.

Bonatti, P. and D. Olmedilla (2005). Driving and monitoring provisional trust negotiation with metapolicies, IEEE.

Bos, N., J. Olson, D. Gergle, G. Olson and Z. Wright (2002). Effects of four computer-mediated communications channels on trust development, ACM.

Brainov, S. and T. Sandholm (1999). Contracting with uncertain level of trust, ACM.

Bratley, P., B. L. Fox and L. E. Scharge (1987). A Guide to Simulation. New York, Springer-Verlag.

Burt, R. S. (1987). "Social contagion and innovation: Cohesion versus structural equivalence." American Journal of Sociology: 1287-1335.

Burt, R. S. and M. Knez (1996). "Trust and third-party gossip." Trust in organizations: Frontiers of theory and 
research 68: 89

Buskens, V. (1995). "Social networks and the effect of reputation on cooperation." ISCORE paper 42.

Buskens, V. (1998). "Network Construction Methods for the Simulation of Stochastic Blockmodels DRAFT."

Buskens, V. (1998). "The social structure of trust." Social Networks 20(3): 265-289.

Buskens, V. (2003). "Trust in triads: effects of exit, control, and learning." Games and Economic Behavior 42(2): $235-252$.

Buskens, V. and W. Raub (2008). "Rational choice research on social dilemmas: embeddedness effects on trust." Handbook of Rational Choice Social Research. New York: Russell Sage.

Buskens, V. and A. Van de Rijt (2008). "Dynamics of networks if everyone strives for structural holes." ajs 114(2): 371-407.

Buskens, V. and K. Yamaguchi (1999). "A new model for information diffusion in heterogeneous social networks." Sociological Methodology 29(1): 281-325.

Buskens, V. W. (2002). Social networks and trust, Kluwer Academic Pub.

Camerer, C. and K. Weigelt (1988). "Experimental tests of a sequential equilibrium reputation model." Econometrica: Journal of the Econometric Society: 1-36.

Coleman, J. S. (1964). "Collective Decisions*." Sociological Inquiry 34(2): 166-181.

Coleman, J. S. (1994). Foundations of social theory, Belknap Press.

Coleman, J. S., E. Katz, H. Menzel and C. U. B. o. A. S. Research (1966). Medical innovation: A diffusion study, Bobbs-Merrill Co.

Dasgupta, P. (2000). "Trust as a Commodity." Trust: Making and Breaking Cooperative Relations, electronic edition, Department of Sociology, University of Oxford: 49-72.

Falcone, R. and C. Castelfranchi (2004). Trust dynamics: How trust is influenced by direct experiences and by trust itself, IEEE Computer Society.

Friedkin, N. E. (1992). "An expected value model of social power: Predictions for selected exchange networks." Social Networks 14(3-4): 213-229.

Friedman, B., P. H. Khan Jr and D. C. Howe (2000). "Trust online." Communications of the ACM 43(12): 34-40.

Fudenberg, D. and J. Tirole (1991). Game theory. 1991, MIT Press.

Gibbons, R. (2001). "Trust in social structures: Hobbes and Coase meet repeated games." Trust in society: 332-353.

Golbeck, J. and J. Hendler (2004a). "Accuracy of metrics for inferring trust and reputation in semantic webbased social networks." Engineering Knowledge in the Age of the SemanticWeb: 116-131.

Goodman, L. A. (1961). "Snowball sampling." The Annals of Mathematical Statistics: 148-170.

Granovetter, M. S. (1973). "The Strength of Weak Ties." The American Journal of Sociology 78(6): 1360-1380.

Harsanyi, J. C. (1995). "A new theory of equilibrium selection for games with complete information."
Games and Economic Behavior 8(1): 91-122.

Harsanyi, J. C. and R. Selten (1988). "A general theory of equilibrium selection in games." MIT Press Books 1.

Haythornthwaite, C. (1996). "Social network analysis: An approach and technique for the study of information exchange." Library \& Information Science Research 18(4): 323-342.

Janssen, M. A. (2011). Small World Networks. Games and Gossip, OpenABM Consortium.

Jarvenpaa, S. L. and D. E. Leidner (1998). "Communication and trust in global virtual teams." Journal of Computer Mediated Communication 3(4): $0-0$.

Kreps, D. M. (1992). "Game theory and economic modelling." OUP Catalogue.

Kreps, D. M. (1996). "Corporate culture and economic theory." Firms, organizations and contracts: a reader in industrial organization: 221-275.

Lia, N., W. Winsborough and J. Mitchell (2003). "Distributed credential chain discovery in trust management." Journal of Computer Security 11(1): $35-86$.

Lipnack, J. and J. Stamps (1997). Virtual teams: Reaching across space, time, and organizations with technology, John Wiley \& Sons Inc.

Mailath, G. J. and L. Samuelson (2001). "Who wants a good reputation?" Review of Economic Studies 68(2): 415-441.

McGuire, W. J. (1966). "Attitudes and opinions." Annual review of psychology 17(1): 475-514.

Nash, J. (1951). "Non-cooperative games." The Annals of Mathematics 54(2): 286-295.

Naylor, T. H., J. Finger, J. L. McKenney, W. E. Schrank and C. C. Holt (1967). "Verification of computer simulation models." Management Science: 92-106.

Porter, R. H. (1983). "Optimal cartel trigger price strategies* 1." Journal of Economic Theory 29(2): 313-338.

Radner, R. (1981). "Monitoring cooperative agreements in a repeated principal-agent relationship." Econometrica: Journal of the Econometric Society: 1127-1148.

Raub, W. and J. Weesie (1990). "Reputation and Efficiency in Social Interactions: An Example of Network Effects." American Journal of Sociology 96(3): 626-654.

Riegelsberger, J., M. A. Sasse and J. D. McCarthy (2003). "The researcher's dilemma: evaluating trust in computer-mediated communication." International Journal of Human-Computer Studies 58(6): 759-781.

Salganik, M. J. and D. D. Heckathorn (2004). "Sampling and Estimation in Hidden Populations Using Respondent Driven Sampling." Sociological Methodology 34(1): 193-240.

Sheskin, D. (2004). Handbook of parametric and nonparametric statistical procedures, CRC Pr I Llc.

Snijders, C. (1996). Trust and commitments, Purdue University Press.

Weesie, J., V. Buskens and W. Raub (1998). The management of trust relations via institutional and 
MEASURING TRUST IN ONLINE SOCIAL NETWORKS - The Effects of Network Parameters on the Level of Trust in Trust Games with Incomplete Information

structural embeddedness. The Problem of Solidarity: Theories and Models. P. Doreian and T. J. Fararo. Amsterdam: 113-138.

Yamaguchi, K. (1996). "Power in networks of substitutable and complementary exchange relations: A rational-choice model and an analysis of power centralization." American Sociological Review: 308332. 